\title{
Contribution of «in situ» NMR to the study of the glucose metabolism of different strains of Fibrobacter species
}

\author{
C Matheron 1, A-M Delort 1, G Gaudet 2,3 ,E Forano 3 \\ ' Laboratoire de Synthèse et Etudes de Systèmes à Intérêt Biologique, URA 485 du CNRS, \\ Université Blaise Pascal, 63177 Aubière; ${ }^{2}$ Centre Universitaire des Sciences et Techniques, \\ Université Blaise Pascal, 63177 Aubière, France; ${ }^{3}$ INRA, Laboratoire de Microbiologie, \\ C.R. de Clermont-Ferrand-Theix, 63122 Saint-Genes-Champanelle, France
}

"In situ» ${ }^{13} \mathrm{C}$ NMR spectroscopy was previously used to study the metabolism of ${ }^{13} \mathrm{C}-1$ glucose by Fibrobacter succinogenes strain $\mathbf{8} 85$ grown on synthetic medium (Gaudet et al, 1992, Eur J Biochem, 207, 155-162). This cellulolytic anaerobic bacteria produces succinate, acetate and formate from glucose catabolism and stores glycogen. It was shown i) that glycogen was simultaneously stored and degraded by the bacterial cells in the presence of exogenous glucose, ii) that part of the glycogen was stored after reversion of the glycolysis at the triose-phosphate level (Gaudet et al, 1992). The patterns of labelling of succinate and acetate synthesized from ${ }^{13} \mathrm{C}-1$ glucose agree well with the pathway of glucose metabolism described by Miller (1978, Arch Microbiol, 117, 145-152), while the percentage of ${ }^{13} \mathrm{C}$ enrichment of these two metabolites, determined by ${ }^{1} \mathrm{H}$ NMR spectroscopy, evidenced a lack of labelling of acetate compared to succinate. This lack of labelling led us to suggest the existence of an alternative pathway of synthesis of acetate. Furthermore, a high phosphoketolase activity was evidenced in 585 cellular extracts (Matheron et al, unpublished results).
Fibrobacter is a genetically diverse yet phylogenetically coherent genus, composed of two species and different groups within the species (Lin and Stahl, 1995, J Bacteriol, 177, 2543-2549). However, there are no physiological traits reflecting this diversity. Furthermore, the strain $\mathrm{S} 85$ of $F$. succinogenes, the most studied strain, was isolated 40 years ago and may have derived since then.

We have undertaken a study of the original metabolic traits found in the strain S85, with other strains of Fibrobacter succinogenes, isolated more recently or belonging to the other groups and with a representative of the $F$. intestinalis species.

Results similar to the one obtained with S85 were obtained with the different strains, allowing to confirm futile cycling of glycogen, and reversion of the glycolysis at the triosephosphate level for all the Fibrobacter strains sudied. The lack of labelling of acetate compared to succinate was also found. The presence of a phosphoketolase activity was evidenced in the cytoplasm of the different strains. These results confirm the apparent physiological homogeneity of the genus Fibrobacter. 